\title{
Aquello es ahora, y esto fue entonces: Walter Lippmann y la crisis del periodismo
}

\author{
Robert W. MCCHESNEY ${ }^{1}$ \\ Traducción de Miguel Álvarez-Peralta \\ miguelalvarez peralta@ccinf.ucm.es \\ rwmcchesney@gmail.com
}

(Abstracts y palabras clave al final del artículo)

Propuesto: 13 de febrero de 2013

Evaluado: 14 de febrero de 2013

Aceptado: 16 de febrero de 2013

Es ampliamente reconocido que Estados Unidos se encuentra hoy sumido en un abrumador colapso del periodismo tal y como lo hemos conocido durante el siglo pasado, si no durante la historia entera de esta nación. El número de periodistas asalariados per cápita ha decrecido gradualmente durante las últimas dos décadas, sufriendo un abrupto desplome a partir de 2007. La publicidad, fuente mayoritaria de ingresos para los medios de información durante el siglo pasado, ha encontrado múltiples opciones nuevas en la era digital, y está en proceso de abandonar el barco. Las plegarias para que las nuevas tecnologías crearan mágicamente un nuevo modelo para un periodismo democrático, suficiente y viable, no están dando resultado. No se avista ningún cambio de rumbo, ni tampoco hay motivos para esperarlo. Los Estados Unidos - de hecho, en cierta medida, todos los países del mundo- enfrentan un problema fundamental: cómo crear medios viables e independientes, o considerar cómo va a ser este mundo sin un cuarto poder.

En The Death and Life of American Journalism (Nation Books, 2010), John Nichols y yo argumentábamos que de la valiosa y a menudo ignorada historia de nuestra prensa, pueden extraerse importantes ideas acerca de cómo generar soluciones a la actual crisis del periodismo. Los padres de la Constitución -principalmente Thomas Jefferson y James Madison-y las primeras generaciones de americanos, no albergaron ninguna ilusión de que el "mercado" fuese a dar lugar a un periodismo adecuado. Ellos destinaron cuantiosas ayudas públicas para generar una prensa libre e independiente, y estos subsidios fueron cruciales para la supervivencia y el crecimiento de la democracia americana y las libertades que tanto valoramos. En estos

\footnotetext{
1 Profesor de la Universidad de Illinois en Urbana-Campaign. Eminente profesor en medios de masas, economía política de la comunicación y en periodismo profesional. Es el editor, con Victor Pickard, del volumen Will The Last Reporter Turn Out the Lights? The Collapse of Journalism and What can be done to prevent it, publicado en 2011, sobre la crisis galopante del periodismo en USA, texto colectivo de donde proviene este artículo, titulado originalmente "That was now, and this is then: Walter Lippmann and the crisis of journalism" Damos las gracias al autor por su gentil permiso para esta traducción.
} 
tiempos en que el mercado muestra poco interés en producir un periodismo adecuado, este es un valioso legado que debemos estudiar y poner en valor. Desde nuestro punto de vista, el futuro de nuestro sistema de autogobierno y nuestra Constitución dependen de ello.

Mientras lidian con la actual crisis, los estadounidenses harían bien en volver la vista al trabajo de Walter Lippmann, uno de los grandes periodistas y más distinguidos intelectuales de la esfera pública del siglo veinte. Aunque durante décadas Lippmann ha ocupado un lugar destacado en los estudios académicos sobre periodismo, en base a sus dos obras clásicas, Public Opinion (1922) y The Phantom Public (1925) $)^{2}$ la elección de Lippmann como referencia para el futuro puede sorprender a algunos estudiosos de la comunicación mediática contemporánea. A partir de estas obras Lippmann ha sido caricaturizado en las últimas dos décadas como alguien escéptico con la democracia popular, si no claramente hostil a la misma. En la versión más extrema, Lippmann es visto como ideólogo de la propuesta de que unos pocos sabios guíen al "rebaño desorientado", así como del empleo de los medios de información para la "fabricación del consenso" en favor de posiciones políticas que servían a los intereses de la élite. Aceptando estos argumentos, la obra de Lippmann debiera ser el último lugar donde indagar por qué y cómo establecer nuevos medios que logren echar leña en el fuego de la democracia.

A pesar de que reconozco el creciente escepticismo de Lippmann hacia la democracia en la década de los años veinte, no creo que una lectura atenta de Public Opinion o The Phantom Public respalde tan bastas generalizaciones. Estos escritos despertaron importantes preocupaciones acerca de los problemas que encaraba la democracia, y de la capacidad del periodismo para generar una ciudadanía autogobernada e informada como postula la teoría democrática. Una parte, quizá la mayoría, de lo que se trata en estos libros - por ejemplo su discusión acerca de la despolitización en la introducción de The Phantom Public- es realmente brillante y ha sobrevivido muy bien hasta nuestros días.

En lo que al periodismo y la información concierne, sin embargo, estos libros no han envejecido especialmente bien. En ambos, el periodismo no es más que un personaje secundario dentro de una trama mucho más amplia. Además, en el contexto histórico de ambas obras el periodismo profesional estaba en ascenso y el sistema comercial de medios de información estaba funcionando. Ante nuestra crisis presente, la perspectiva adoptada por Lippmann no se adecua especialmente bien a las grandes cuestiones que encaramos en el ámbito del periodismo.

Es por ello una suerte considerable para nosotros que en 1920 Lippmann escribiera dos textos bastante desconocidos, que tratan directamente el tema del periodismo y su relación con el autogobierno. Estos son: "A Test of the News", un largo ensayo del que Charles Merz es coautor, aparecido en el New Republic en agosto de 1920; y Liberty and the News ${ }^{3}$ (1920), un libro de tres capítulos, dos de los cuales fueron publicados en el Atlantic Monthly. "A Test of the News" analiza de forma sis-

\footnotetext{
2 N.d.T: Traducidos al español como La Opinión Pública y El Público Fantasma, respectivamente.

${ }^{3}$ N.d.T: Traducida al español como Libertad y Prensa.
} 
temática la cobertura de la Revolución Rusa realizada por el New York Times entre 1917 y 1920. Se trata de una investigación pionera que se anticipaba en décadas a algunos de los mejores análisis de contenido de los tiempos recientes. Liberty and the News es el único encuentro directo de Lippmann con la relación entre periodismo, democracia y libertad. Contiene ejemplos de su prosa más admirable, y un trabajo extraordinario para cualquier criterio de valoración, que incorpora numerosas dimensiones analíticas. No se puede leer estos trabajos y descartar a Lippmann como un elitista antidemocrático.

Estos trabajos cobran incluso mayor relevancia porque fueron escritos coincidiendo con el punto álgido de la verdadera última crisis de definición para el periodismo. Un número desproporcionadamente alto de los más grandes progresos en ciencias sociales tiene lugar en tiempos de crisis y agitación, cuando las formulaciones que se tienen por comprobadas son sometidas a mayor escrutinio. (Tómese a John Maynard Keynes y la Gran Depresión como ejemplo). Enfrentados a una crisis, nuestros mejores pensadores se tornan a menudo más críticos, creativos y originales, proporcionándonos enseñanzas y nociones que perviven a lo largo de los años. De igual modo, este periodo específico fue un tiempo extraordinariamente fecundo para el desarrollo y la cristalización del pensamiento de Lippmann acerca de la prensa y el autogobierno. Con el cambio de los tiempos, su trabajo se movería rápidamente en una dirección diferente, políticamente más conservadora. Se desplazó de las preocupaciones estructurales hacia un mayor énfasis en la psicología humana como variable independiente fundamental, tomando las instituciones sociales prácticamente como dadas; pero eso no altera la potencia ni la importancia de lo que escribió en 1919 y 1920. Lippmann, de hecho, nunca renegó de la investigación y las posiciones recogidas en "A Test of the News" o Liberty and the News, incluso si realmente nunca las retomó a través de la pluma ni en espíritu. Éstas pertenecieron a un momento concreto.

A menudo pasa desapercibido para observadores contemporáneos cómo en 1919 y 1920 la credibilidad y legitimidad de las noticias era ampliamente cuestionada por la mayor parte del público americano. "Es admitido que una saludable opinión pública no puede existir si no existe acceso a la información", afirmaban Lippmann y Merz. "Existe hoy una extendida y creciente duda acerca de la existencia de tal acceso a la información sobre asuntos polémicos. Esta duda varía desde acusaciones de sesgo inconsciente, hasta denuncias directas por casos de corrupción, desde la creencia de que la información ha sido maquillada, hasta la creencia de que la información está envenenada" (p.1). El principal factor, aunque no el único, que explicaría la amenaza sufrida por la información y la democracia, fue la fulminante emergencia de la propaganda organizada, aquello a lo que hoy nos referimos frecuentemente como relaciones públicas. Durante la Primera Guerra Mundial, Lippmann conoció de primera mano el uso exitoso de este tipo de propaganda por el gobierno de los Estados Unidos, y por una prensa complaciente con el moldeado de la opinión pública. Lippmann señaló que la propaganda empeoró con creces el ya "extremadamente reaccionario" y "crecientemente perjudicial" periodismo comercial de aquellos tiempos (p.29).

En ningún sitio quedaba esto más patente que en el detallado análisis que Lippmann y Merz hicieron sobre la cobertura que el New York Times dio a la 
Revolución Rusa. A pesar de sus escasas simpatías por la revolución, ambos quedarían espantados ante lo que encontraron: básicamente noticias que reportaban como verdades sagradas los deseos, distorsiones y mentiras de las fuerzas contrarrevolucionarias. El mayor mentiroso sería el propio gobierno de los Estados Unidos. El Times estaba siendo "seriamente engañado" por "su confianza en los proveedores oficiales de información" (p.41). Su conclusión afirmaba: "La cobertura de la Revolución Rusa es un absoluto desastre. El resultado final es casi siempre engañoso en las cuestiones esenciales, y una noticia engañosa es peor que ninguna noticia". Los periodistas "estaban ejecutando el deber democrático supremo de suministrar la información que alimenta la opinión pública, y fueron negligentes en esta misión[...] Cualesquiera que sean las excusas, las disculpas, los atenuantes, permanece el hecho de que no se le pudo garantizar la información mínima necesaria sobre un evento de extraordinaria importancia a un gran pueblo inmerso en una crisis colosal". (p.3).

Lippmann hizo énfasis en el modo en que la propaganda gubernamental había alterado la ecuación democrática tradicional. Encontró el aumento de esta propaganda realmente aterrador, generador de una crisis existencial para la noción misma de prensa libre, y por tanto para la de autogobierno: "el gobierno tiende a operar en función del impacto que una opinión controlada tiene sobre la administración. Este desplazamiento en el locus de la soberanía ha hecho que se priorice la fabricación del consenso[...] Sin una protección contra la propaganda, sin estándares para la demostración de hechos, sin criterios para el énfasis, la sustancia viva de toda decisión popular queda expuesta a todos los prejuicios y a un abuso infinito.” (p.37).

Las implicaciones de la corrupción y degradación de la información no podrían ser más graves. Para Lippmann, el pueblo está cada vez más "desconcertado porque los hechos no están disponibles; y se pregunta si el gobierno democrático puede sobrevivir en un tiempo en el que la fabricación del consenso es asunto de empresas privadas no sujetas a regulación. Porque en un sentido preciso, la presente crisis de la democracia occidental es una crisis del periodismo" (p.2, énfasis mío). Así pues, los medios de información eran instituciones de singular relevancia. "El periódico es literalmente la biblia de la democracia, el libro a partir del cual la ciudadanía determina su conducta[...] Ahora, el poder para determinar cada día qué será considerado importante y qué será obviado es un poder distinto a cualquier otro poder que haya sido ejercido desde que el Papa perdió su dominio sobre la mentalidad laica" (p.28). De ahí lo que Lippmann y Merz consideraran "una tarea fundamental del siglo veinte: asegurar a un pueblo libre un suministro de noticias tal que un gobierno libre pueda ser administrado con éxito" (p.3).

Lo que resulta notable en estos trabajos es la convicción de que el periodismo y las instituciones que lo producen no deben ser pensados como empresas privadas sino como instituciones públicas. "Un buen periódico es una institución de servicio público", escribieron Lippmann y Merz. "Ocupa una posición en la vida pública tan importante como el sistema educativo, la iglesia, o los órganos de gobierno" (p.4). Lippmann hizo notar que "Las columnas periodísticas son canales públicos. Cuando aquellos que las controlan se arrogan el derecho a determinar según su propia consciencia qué debe ser reportado y con qué propósito, la democracia es impracticable" (pp.5-6). 
Resumiendo, ciudadanía y gobierno tienen un interés directo en vigilar que el sistema de medios informativos funcione correctamente, y los propietarios no tienen derecho a reclamarlo como una propiedad privada ajena al interés público. En Liberty and the News, se sugiere que podría gastarse dinero público en mejorar la calidad de la información.

En 1920, Lippmann no estaba en absoluto seguro de que esta crisis fuese a resolverse de forma satisfactoria. "En pocas generaciones, a los historiadores les parecerá absurdo que una nación que proclama gobernarse según la voluntad del pueblo no debiera hacer esfuerzos por garantizar una información sin la cual es imposible formar una opinión para gobernarse. 'Cómo puede ser,' se preguntarán, 'que a comienzos del siglo veinte, naciones que se proclamaban democráticas se contentasen con actuar según lo que casualmente pasase por la puerta; que aparte de unos pocos llamamientos y protestas esporádicas no se hiciera ningún plan para poner esos canales públicos bajo control social'” (p.8).

Lippmann mantuvo una actitud ambigua hacia el sistema de medios de información comerciales y los propietarios de periódicos. Las primeras dos décadas del siglo veinte constituyeron una crisis existencial para ellos, dado que el partidismo descarado, el sensacionalismo, la corrupción y los escándalos minaron la legitimidad del sistema comercial. Algunos editores llegaron a temer por su existencia. Lippmann reconoció esta amenaza y mostró poca simpatía por la mayoría de los propietarios: "De uno otro modo, la próxima generación intentará poner el negocio editorial bajo un mayor control social. Por todas partes aparece un desencanto cada vez más molesto con la prensa, una creciente sensación de estar siendo manipulados y confundidos." Lippmann concluyó que "algún día el Congreso, en un ataque de ira, alentado por una opinión pública furiosa, intervendrá sobre la prensa con un hacha" (p.45). Él ni siquiera contempló la noción de que la competencia en un "mercado libre" fuese a producir por voluntad propia el periodismo necesario para que el autogobierno progresase. Aun siendo enérgico opositor a la censura, Lippmann no mostró preocupación alguna por cómo una intervención pública podría violar aquellos derechos que la Primera Enmienda reconoce a los editores.

Dicho esto, Lippmann no consideraba a los propietarios de periódicos como una infranqueable barrera estructural a efectos de reformas. En sus análisis, asumía constantemente que el poder real para controlar la información quedaba en los jefes de redacción, no en los editores. Tenía poco interés por cómo los intereses políticos o comerciales de los propietarios determinaban o influenciaban quiénes llegaban a dirigir las publicaciones, y qué valores guiaban su trabajo. Lippmann tenía aparentemente poco interés en la connivencia entre gobiernos y grandes empresas, ni tampoco se mostró especialmente preocupado por cómo el periodismo afectaba a diferentes sectores de la población. No todos los que quedaban fuera de las altas esferas de gobierno salían perdiendo si las noticias fracasaban en su misión de crear una ciudadanía informada y participativa. Sorprendentemente para alguien que había sido un socialista tan solo unos años antes, nunca cultivó la idea, fundamental tanto para Jefferson como para Madison, de que quienes detentaban propiedades y privilegios se beneficiarían enormemente de un pueblo ignorante y mal informado. No tenía, para decirlo sin rodeos, ningún análisis de clase. 
Casi al mismo tiempo que se publicaban Liberty and the News y "A Test of the News", veía la luz también el trabajo de Upton Sinclair, The Brass Check. Sinclair denostó a los editores de periódicos por la corrupción y el sesgo anti-obrero de sus informaciones. El libro está repleto de ejemplos, y hace imposible contemplar la propiedad efectiva de los medios como algo irrelevante para el funcionamiento de las redacciones o el contenido de las noticias, como Lippmann presupone. La obra tuvo además una fuerte repercusión en la historia del periodismo. Pero Sinclair utilizó un martillo en tiempos en que el escalpelo de Lippmann hubiera sido preferible. Ambos se hubieran beneficiado enormemente de un encuentro con la obra del otro. Desdichadamente, se criticaron uno a otro sin más, probablemente debido a diferencias políticas, como prueba la ligereza con que Lippmann desdeña The Brass Check en Public Opinión (pp. 182-83).

Incluso un socialista como Sinclair tenía dificultades para imaginar que un sistema de medios de información diferente al modelo de propiedad privada pudiera tener lugar en Estados Unidos, de modo que difícilmente podemos recriminar a Lippmann por internalizar como "dado" el sistema comercial dominante no solo en aquella época, sino durante todo el siglo. Contar con buenos editores, era por tanto considerados posible y necesario. Lippmann dedicó Liberty and the News a C.P. Scott, el legendario propietario y editor del Manchester Guardian desde 1872. "A la luz de su carrera," escribió Lippmann, "no pueden parecernos absurdas ni remotas las ideas de libertad y verdad en relación a la información" (p.XXV). Quizá sea un indicador de la importancia de las presiones comerciales sobre la información periodística el hecho de que, tras la muerte de Scott, su familia dejara The Guardian en manos de una fundación sin ánimo de lucro, a partir de 1936. El principio fundacional declarado por esta organización era "salvaguardar la independencia financiera y editorial de The Guardian en el futuro, mientras los objetivos subsidiarios son la defensa de sus principios y la promoción de la libertad de prensa en el Reino Unido y en el extranjero" (McChesney y Nichols, p. 176). (Esta estrategia de renuncia al lucro ha dado buenos resultados al periódico. Mientras los medios de información comerciales naufragan en la presente crisis, puede argumentarse desde muchos puntos de vista que The Guardian constituye todavía, hoy mejor y con más importancia que nunca, una fuente de periodismo de calidad, ahora además también en el terreno digital).

En Liberty and the News, Lippmann no estaba dando el paso radical de abogar por la creación de periódicos sin ánimo de lucro; en vez de eso, estaba en primer lugar poniendo sobre aviso a los propietarios para que se percataran de que debían cambiar de rumbo. Esperaba que el miedo a la extinción alentara a los editores con menos principios que Scott a que cumpliesen con su deber. "Los editores sabios no desdeñarán estos presagios," escribió. "La regulación del negocio editorial es de carácter sutil y elusivo, y solo mediante un esfuerzo rápido y comprensivo para combatir sus males pueden las mentes más sensatas mantener el control" (p.45).

La solución era clara para Lippmann, y en esto hizo mucho énfasis: los periódicos necesitaban adoptar una formación y estándares profesionales para reporteros y editores que aseguraran la más alta calidad de información, precisa en términos fácticos y honestamente contextualizada, libre de sesgos personales, comerciales o polí- 
ticos. "En primer lugar," escribieron Lippmann y Merz, "creemos que los estándares profesionales del periodismo no son lo suficientemente elevados, y la disciplina por la que dichos estándares se mantienen no es suficientemente fuerte" (p.41). La labor informativa, según Lippmann, debía mantenerse alejada de "manos tendenciosas sin preparación"(p.60). "La actual estructura informativa debe transformarse en un servicio a la democracia, siguiendo las líneas generales aquí sugeridas, mediante la formación del periodista y la implantación del registro y análisis expertos" (p.59).

"¿Hasta dónde podremos llegar en la transformación de la empresa periodística desde un descuidado negocio hacia una disciplinada profesión?" se preguntaba Lippmann. "Bastante lejos, supongo, porque al fin y al cabo es impensable que una sociedad como la nuestra pueda depender por siempre de testimonios fortuitos e inexpertos". (p.46). Hacía falta un compromiso por parte de los dueños de los periódicos y de los propios periodistas, así como financiación pública para instituciones como escuelas de periodismo que formasen a una generación de buenos reporteros con experiencia. "No hay cantidad de dinero y esfuerzo empleados en preparar a los hombres adecuados para este trabajo que puedan considerarse malgastados, porque la salud de la sociedad depende de la calidad de la información que recibe" (p.47).

Lippmann no albergaba ilusiones respecto a que la crisis que afligía al periodismo en 1920 pudiera ser resuelta internamente por propietarios y editores, o simplemente consultando a la élite intelectual. Él consideraba la implantación de estándares profesionales como algo necesario pero no suficiente, y difícilmente realizable de forma satisfactoria sin participación popular. Resulta llamativa la insistencia de Lippmann -acusado a menudo de elitismo- en la importancia del rol manifiesto y permanente que el público debía jugar en la creación de un sistema de medios de información adecuado. "Muy raramente los hombres de la prensa muestran confianza en el público general. Se verán obligados a ello tarde o temprano," afirmó. "La filosofía misma de la profesión debe ser debatida; las noticias sobre las noticias deben contarse. Porque la información sobre la gestión de la estructura informativa toca el núcleo de todo gobierno moderno" (p.9). El debate sobre el periodismo debía darse ante el público general; era asunto de todos. Si se delegaba en las élites y en los involucrados, la reforma sería poco probable: "Quienes ahora tienen el control albergan demasiados intereses en juego, y controlan la fuente misma de reformas." Particularmente importante sería para los progresistas políticamente organizados tomarse la cuestión del periodismo con seriedad: "el cambio llegará únicamente por la rivalidad enérgica de aquellos cuyos intereses no están representados en el actual orden informativo. Llegará solo si la fuerza de trabajo organizada y el liberalismo militante fijan una ruta que no pueda ser ignorada" (p.60).

La primera tarea para el reportero experto y formado sería ver a través de la propaganda y el sesgo originados por gobiernos e intereses poderosos, o por las propias opiniones del reportero, para que los ciudadanos puedan recibir una visión precisa de los acontecimientos del día: "Llegando más allá de la opinión, hasta la información en que esta se basa, y haciendo de la validez de las noticias nuestro ideal, estaremos luchando donde realmente está la batalla. Estaremos protegiendo para el interés público aquello que todos los intereses especiales del mundo están más ansiosos por corromper" (p.41). 
En la visión de Lippmann y Merz, los reporteros necesitan crear y hacer cumplir un "código de honor" profesional, similar al que adoptan y ejecutan las asociaciones profesionales de abogados o médicos, y además deben "observar vigilantes toda infracción a dicho código" (p.42). El giro hacia el profesionalismo, argumentaba Lippmann, podría crear un "gran periodismo independiente que fije los estándares para el periodismo comercial". En resumen, la profesionalización podría forzar incluso a los editores más recalcitrantes a plegarse, del mismo modo que las "espléndidas sociedades cooperativas inglesas" habían fijado unos estándares elevados para las empresas comerciales (p.60).

A partir de estos escritos, podríamos reconocer en Lippmann al eminente visionario que mejor imaginó el rol que la profesionalización llegaría a jugar, rescatando al periodismo del déficit de credibilidad que sufrió durante las dos primeras décadas del siglo veinte. Este logro histórico no es baladí. A principios de la década de los veinte, ya se había creado la Sociedad Americana de Editores de Periódicos y entre sus primeras tareas figuraba establecer un código de conducta. Las escuelas de periodismo, apenas existentes antes de la Primera Guerra Mundial, se multiplicaban y adquirían gran relevancia. Éste sería el principal avance del periodismo para la siguiente generación, y los límites precisos del código profesional fueron debatidos por reporteros, editores y propietarios. Para la década de los cincuenta quedaban ya pocos periódicos abiertamente partidistas, puesto que la mayoría de las redacciones operaban bajo los principios profesionales. Incluso aquellos que durante más tiempo mantuvieron su filiación, como el Chicago Tribune, abrazaron la noción de profesionalismo e imparcialidad periodística -tras la muerte del Coronel Robert McCormick- y una "separación iglesia-estado" entre el departamento de negocios y la oficina editorial. La noción de que la información debería ser imparcial y "objetiva" se convirtió en un lugar común, hasta el punto de que generaciones enteras de estadounidenses entendieron que ésta era la intención de la Primera Enmienda.

No deberíamos llevar la idea de Lippmann como "padre fundador" del periodismo profesional americano demasiado lejos, puesto que la forma en que el periodismo se profesionalizó en Estados Unidos diverge considerablemente de los valores fundamentales por él prescritos en Liberty and the News y en "A Test of the News". Lo más notable, es que Lippmann y Merz pensaron que la clave principal en el asunto del periodismo profesional era que fuese capaz de diseccionar rigurosamente, contrastar, contextualizar y criticar la información proveniente de gobiernos y fuentes interesadas. "Declaraciones provenientes de gobiernos o círculos cercanos al gobierno no pueden ser tomadas como hechos comprobados por la prensa independiente" (p.41). Resulta irónico que una tendencia principal del periodismo profesional, tal y como ha evolucionado en Estados Unidos, haya sido tomar declaraciones del gobierno al pie de la letra, especialmente si existe sintonía entre quienes están en el poder, aun cuando hay enormes fundamentos para el escepticismo. La espantosa cobertura de los antecedentes de la invasión estadounidense de Iraq en 2003 es una buena muestra de ello, pero la lista es larga y deprimente. La información periodística contemporánea suspende el punto principal en el test de Lippmann para cualquier valoración honesta. 
Aunque Lippmann estuvo obsesionado con lograr periodistas bien entrenados haciendo periodismo objetivo, nunca pensó que tal información objetiva fuese a acabar con las discusiones políticas o pudiera minar la opinión política. Simplemente pensó que debería ser su base. No se opuso en absoluto al periodismo de opinión -después de todo, fue cofundador del New Republic y, durante muchas décadas, un premiado columnista político-, su razonamiento era más bien que el periodismo de opinión requería de una gran cantidad de noticias dedicadas primeramente a suministrar una comprensión honesta, por oposición a otra guiada por motivaciones partidistas o comerciales.

Podemos debatir si tal periodismo realmente objetivo puede llegar a existir, y de hecho pocos creen que sea posible. Los sesgos institucionales y humanos son inevitables, y el punto de partida es ser honestos al respecto. Pero esto no invalida la preocupación de Lippmann. Cierto periodismo puede ser "más" objetivo que otro, y necesitamos un periodismo que trate de hacerlo lo mejor posible, manteniéndose responsable con respecto al cumplimiento de ese estándar. Quizá la mejor manera de expresarlo es que necesitamos que los periodistas sean investigadores, no abogados. Los investigadores académicos, si son honestos, buscan las críticas a sus propios argumentos, así como evidencias en contra, y tratan de adquirir la comprensión más fuerte y precisa posible de su materia. Los abogados, en cambio, solo pretenden ganar. Seleccionan interesadamente hechos y declaraciones, y obvian el contexto cuando éste va en contra de la posición que defienden. Ellos solo tratan con las posiciones opuestas y las evidencias incómodas si se ven obligados, y en este caso la clave está en desacreditar o minimizar el impacto empleando cualquier estrategia necesaria. El periodismo de Lippmann es un periodismo de investigadores. El periodismo de abogados no es en absoluto periodismo democrático.

En el mundo que vivimos, demasiados aspectos de la vida pública quedan infrarrepresentados por los periodistas, de modo que las advertencias de Lippmann sobre las consecuencias de la falta de información basada en hechos se tornan más y más amenazantes: "El punto cardinal es siempre la pérdida de contacto con la información objetiva. La razón pública depende de ello tanto como la privada. No lo que alguien dice, tampoco lo que alguien desea que sea cierto, si no lo que está más allá de nuestras opiniones, constituye el criterio de nuestra cordura" (p.34). "Lo realmente importante," añadió, "es intentar hacer que la opinión sea cada vez más una respuesta a los hechos. No puede haber libertad para una comunidad que carece de la información con la que detectar mentiras" (p.38). Nuestras libertades fundamentales pierden la base sobre la que se asientan. "Cuando la libertad de opinión se expresa como libertad de error, ilusión y malinterpretación, es virtualmente imposible suscitar mucho interés por ella. Es la más pobre de las abstracciones y una sofisticación meramente intelectual" (p.39). "No parece haber modo alguno de evadir la conclusión," indicaba Lippmann, "de que la libertad no está tanto en la autorización como en la construcción de un sistema informativo crecientemente independiente de la opinión" (p.57).

Un mundo sin periodismo y atestado de gente libre para esparcir sus opiniones, por tanto, difícilmente es una sociedad libre, y no puede ser tenido por democrático en sentido alguno. Se trataría más bien de un infierno en la tierra: "Hombres que han 
perdido contacto con los hechos relevantes de su entorno son víctimas inevitables de la agitación y la propaganda. Brujos, charlatanes, extremistas y terroristas florecen allí donde una audiencia es privada del acceso independiente a la información." (p.32). Lippmann fue contundente acerca del rumbo de un futuro sin periodismo libre: "Estoy igualmente convencido de que la democracia degenerará en esta dictadura bien de la derecha o de la izquierda, si no alcanza un autogobierno genuino" (p.59).

Hoy estamos inmersos en una crisis del periodismo y de la democracia no menos sobrecogedora que la de hace noventa años. Hasta cierto punto, responde a la degradación del periodismo profesional, causada en gran medida (aunque no exclusivamente) por las fuerzas mercantiles que Lippmann no tuvo en cuenta en su análisis. El enérgico e intrépido periodismo independiente que Lippmann deseaba resultó incompatible con un sistema de medios informativos comercial y considerablemente monopólico. En un plano todavía más fundamental, la revolución tecnológica ha desintegrado el modelo de negocio del periodismo comercial, dejando lo que de él quedaba sumido en una gran incertidumbre. Ya no existe la base de recursos que según Lippmann podría financiar las redacciones periodísticas de la nación.

Eso significa que no podemos usar el guión de Lippmann de los años veinte para determinar la solución exacta a la presente crisis. Bien, ¿y qué? No podemos usar necesariamente las mismas políticas y subsidios que Jefferson o Madison, pero aun así ellos, al igual que Lippmann, tienen lecciones cruciales para nosotros sobre democracia y periodismo, como también Lippmann las tiene. Su visión del papel principal que prensa e información tienen para la libertad y la democracia es verdaderamente sublime. Su comprensión de la naturaleza pública y política del periodismo y del papel crucial que desempeña el público es una réplica necesaria al absurdo y anti-histórico fundamentalismo de mercado que ha hecho descarrilar nuestro debate sobre la crisis del periodismo. Su crítica de una "libertad" fatua, que sirva básicamente para mentir con impunidad en un mundo sin hechos, un mundo de tergiversación y propaganda, debería producir escalofríos a lo largo de nuestra espina dorsal. Tenemos ya un pie en esa realidad, y no podemos permitirnos esperar y ver qué pasa en el caso de que el otro pie aterrice también en ella.

Resolver la crisis del periodismo, crear un cuarto poder independiente que haga verdaderamente posible el auto-gobierno y la libertad, será difícil, pero no es imposible. Fue la misión de los creadores de nuestra Constitución, fue la misión de Lippmann, y debe ser la nuestra. Quizá no alcancemos la solución perfecta, pero podemos llegar a soluciones mucho mejores que si no hacemos nada salvo mantener el orden actual. En Liberty and the News, Lippmann encontraba razones para el optimismo en nuestra naturaleza esencial: "El deseo de saber, la aversión a ser engañados y manipulados, es una motivación realmente poderosa, y es esa motivación la que mejor puede reclutarse en favor de la causa de la libertad" (p.39). No podemos permitirnos olvidar la enseñanza fundamental de Lippmann: que en un sentido preciso, la actual crisis de la democracia es una crisis del periodismo. 


\title{
RESUMEN
}

Ante la actual crisis del periodismo, McChesney busca respuestas en dos obras no muy conocidas del consagrado Walter Lippmann, escritas durante la grave crisis democrática de 1920: el artículo "A test of the News" y el libro Liberty and the News. En estos textos, Lippmann defiende un acceso suficiente a información de calidad para logar una opinión pública saludable, como base de todo sistema democrático. Ello autoriza a considerar el periodismo un servicio público que no puede abandonarse a las veleidades del mercado o a la presión gubernamental, y promueve la inversión pública para facilitar la aparición de medios independientes.

Palabras clave: Walter Lippman, periodismo, crisis, autogobierno, función social de la prensa

\begin{abstract}
In the midst of a collapse of traditional journalism, McChesney recovers two largely unknown pieces from Walter Lippmann, written during a democratic crisis in 1920: the essay "A test of the News" and the book Liberty and the News. In these works, Lippmann reclaims a sufficient access to quality news in order to produce a sound public opinion, which is the base of self-government and democracy. Therefore, journalism should be considered as a public service that can not simply be left out to pressures from the market or governments. This vision encourages public funding for the development of an independent media information system.
\end{abstract}

Keywords: Walter Lippman, journalism, crisis, self-government, social function of the press

\section{RÉSUMÉ}

Devant la crise actuelle dans le journalisme, McChesney cherche des réponses dans deux petites œuvres connues du légéndaire auteur Walter Lippmann, écrites pendant la crise démocratique de 1920: Liberté pour la Presse et Un text pour les Nouvelles. Dans ces textes, Lippmann défend un accès suffisant à l'information de qualité pour atteindre l'opinion publique en bonne santé, comme la base de tout système démocratique. Cela permet de considérer le journalisme comme un service public qui ne peut pas être laissé aux aléas du marché ou de la pression du gouvernement, et favorise l'investissement public afin de faciliter l'émergence de médias indépendants.

Mots clé: Walter Lippman, crise du journalisme, autonomie, fonction sociale de la presse 